\title{
INMIGRACIÓN, EMIGRACIÓN Y TRÁNSITO MIGRATORIO EN CHIAPAS: UN BOSQUEJO GENERAL
}

\author{
María Eugenia Anguiano Téllez
}

Resumen: En este trabajo se explora de manera general y panorámica la condición actual del estado de Chiapas como territorio de inmigración nacional y extranjera, como entidad expulsora de migrantes al resto del país y Estados Unidos, y como espacio y trayecto de tránsito migratorio mayoritariamente de guatemaltecos pero también de centroamericanos y migrantes de otras nacionalidades. Para ello, se analiza información que proporcionan el Instituto Nacional de Geografía, Estadística e Informática, el Consejo Nacional de Población, el Instituto Nacional de Migración y dos encuestas de flujos migratorios en las que participa El Colegio de la Frontera Norte. Si bien este escrito se limita a presentar un bosquejo de la dinámica migratoria reciente del estado de Chiapas, tiene la pretensión de motivar el interés de investigaciones futuras sobre un tema de creciente relevancia social, económica y política para la entidad y su población.

Palabras clave: Chiapas, inmigración, emigración, transito migratorio, migración laboral.

Enviado a dictamen: 06 de agosto de 2008. Aprobación: 17 de octubre de 2008.

Dra. María Eugenia Anguiano Téllez, doctora en Ciencia Social con especialidad en Sociología por El Colegio de México, labora en El Colegio de la Frontera Norte, temas de especialización: Migraciones interna e internacional, teléfono: (664) 631-6300 extensión 3203, fax: (664) 631-6312, correo electrónico: anguiano@colef.mx, maruanguiano@gmail.com.
Abstract: In this paper, I present a general overview related to the recent circumstances of the state of Chiapas in three aspects: as a territory of immigration, both national and foreign; as anentity to push out migrants to the rest of the country and the United States, and as space and transit route for migratory flows, mostly coming from Guatemala, but also from other Central American countries. In order to do this, I analyze information provided by the National Institute of Geography, Statistics and Informatics; the National Population Council; the National Institute of Migration, and two surveys of migratory flows make available by $\mathrm{El} \mathrm{Colegio} \mathrm{de}$ la Frontera Norte and other Institutions. Even though this paper was merely present an outline of the recent migration of the state of Chiapas, it has a claim to motivate the interest of future research on a topic of increasing relevance social, economic and political for Chiapas and its people.

Key Words: Chiapas, immigration, emigration, migration in transit, labor migration.

\section{Introducción}

$\mathrm{H}$ istórica y culturalmente, Chiapas ha sido un espacio social habitado por diversidad de grupos étnicos y lingüísticos que tanto la corona española como el estado mexicano trataron continuamente de incorporar e integrar a sus dominios colonial y 
nacional. Hace más de un siglo que el Estado de Chiapas forma parte de la frontera sur de México, que colinda con Guatemala y por extensión con Centroamericana. Desde su incorporación a la nación mexicana, procesos migratorios diversos y complejos por su intensidad, composición, volumen y modalidades han marcado su dinámica poblacional (Castillo, 2003). La política de colonización mexicana de finales del siglo XIX y principios del XX estimuló la inversión de capital extranjero y propició el asentamiento de inmigrantes, colonos y trabajadores en territorio chiapaneco, inversionistas y colonos norteamericanos, alemanes y japoneses y trabajadores chinos, guatemaltecos e indígenas de la propia entidad se movilizaron hacia la región del Soconusco (Tovar, 2000; CONAPO, 2000; Casillas, 2008). Al igual que ocurrió en otras regiones del país, en la década de los años 1940 también a Chiapas llegaron refugiados españoles y realizaron valiosas aportaciones a la vida intelectual y cultural de la entidad (Morales, 2003). De 1940 a 1980, el reparto agrario promovió la ocupación y colonización de tierras vírgenes en la Selva Lacandona y la Zona Fronteriza, con familias procedentes del mismo estado y otros del sur y centro del país - Veracruz, Tabasco, Oaxaca, Michoacán, Estado de México, Tlaxcala e Hidalgo- (Cruz y Robledo, 2001). Desde la década de los años 1970, en la entidad chiapaneca ocurrieron desplazamientos masivos de población asociados tanto a procesos de industrialización y urbanización (desarrollo de proyectos petroleros e hidroeléctricos, expansión de la ganadería comercial, crecimiento de sus principales centros urbanos y desarrollo turístico), como a conflictos políticos y religiosos que se extienden hasta la actualidad (Del Rey, 1998; Guillén, 2003; Olivera, 2004; Martínez, 2005). Durante la década de los años 1980, los conflictos armados en Centroamérica generaron un significativo éxodo poblacional de ese subcontinente hacia territorio mexicano, particularmente hacia las entidades colindantes de su frontera sur (Castillo, 1994; Kauffer, 2005). En años más recientes, varias investigaciones han dado cuenta de la emigración de los residentes y nativos chiapanecos, especialmente de aquellos que se desplazan fuera de la entidad y del país (Peña et al., 2000; Villafuerte y García, 2004; Burke, 2004; Peña, 2005; García et al., 2007, Jáuregui y Ávila, 2007; Chávez, en prensa).

Parafraseando una sugerente afirmación de Daniel Villafuerte (2007), en la que postula que "los espacios se hacen visibles en la medida en que en ellos ocurren fenómenos que se vuelven noticia", podemos considerar que la dinámica migratoria de la entidad chiapaneca se ha vuelto noticia y su territorio espacio visible de expulsión, recepción y tránsito de flujos migratorios. En años recientes se ha advertido una creciente participación de los chiapanecos en movimientos migratorios de larga distancia, especialmente reconocidos a partir de las cifras registradas por fuentes oficiales respecto al monto de remesas enviadas desde el exterior de la entidad y del país. ${ }^{1}$ A la par, Chiapas se ha ubicado como una entidad de inmigración y tránsito migratorio para trabajadores centroamericanos, mayoritariamente guatemaltecos, que se dirigen tanto a su territorio como a otras entidades mexicanas y los Estados Unidos, básicamente en busca de oportunidades de empleo.

Siguiendo el sugerente postulado de Villafuerte, en este trabajo se explora de manera general y panorámica la condición actual del estado de Chiapas como territorio de inmigración nacional y extranjera, como entidad expulsora de migrantes al resto del país y Estados Unidos, y como espacio y trayecto de tránsito migratorio, mayoritariamente de guatemaltecos pero también de centroamericanos y migrantes de otras nacionalidades. Para ello, se analiza información que proporcionan el Instituto Nacional de Geografía, Estadística e Informática, el Consejo Nacional de Población, el Instituto Nacional de Migración y dos encuestas de flujos migratorios en las que participa El Colegio de la Frontera Norte. Si bien este escrito se limita a presentar un bosquejo de la dinámica migratoria reciente del estado de Chiapas, tiene la pretensión de motivar el interés de investigaciones futuras sobre un tema de creciente relevancia social, económica y política para la entidad y su población. 


\section{Panorama reciente de los flujos y circuitos migra- torios en Chiapas}

De acuerdo con información del Consejo Nacional de Población (2005: 56), en el contexto nacional y en el año 2005, Chiapas ocupó el segundo lugar por su índice y grado de muy alta marginación, aunque algunos de sus indicadores socioeconómicos la sitúan en un primer sitio; entre ellos podemos anotar que cerca de $60 \%$ de los chiapanecos residen en localidades menores a 5 mil habitantes (58.46\%); la quinta parte de su población es analfabeta (21.32\%); cuatro de cada diez habitantes de 15 años de edad y mayores no concluyeron su educación primaria $(42.76 \%)$ y poco más de tres cuartas partes de su población ocupada recibía un ingreso menor a dos salarios mínimos mensuales (78.14\%). A pesar de la desigual distribución del ingreso que priva en la entidad y que la ubica en el contexto nacional en los primeros lugares de marginación económica y social, la explotación de sus recursos naturales y su estratégica industria hidroeléctrica, sus tradicionales explotaciones agrícolas, ganaderas y forestales y el desarrollo de sus sectores comercial y de servicios, especialmente el turístico, han conformado un amplio mercado laboral que demanda trabajadores tanto residentes en la entidad como procedentes de territorios circunvecinos del país y del extranjero, estos últimos tradicional y mayoritariamente guatemaltecos.

$\mathrm{Al}$ interior de lo que hoy conforma la entidad chiapaneca, aún antes de su integración como territorio mexicano, ocurrían movimientos de carácter laboral de personas que se desplazaban hacia sus localidades o a través de ellas para emplearse principalmente en actividades del sector primario (Castillo, 2003). Desde finales del siglo XIX y durante todo el siglo XX, las plantaciones cafetaleras de la región Socunusco han empleado trabajadores procedentes de otras regiones del estado, de otras entidades mexicanas aledañas y de otros países, especialmente de Guatemala (Tovar, 2000; Villafuerte y García, 2004). En las décadas de los años 1970 y 1980, la construcción de grandes presas hidroeléctricas y la explotación petrolera tuvieron consecuencias múltiples en la población y el empleo. En primer término, desplazaron masivamente poblados que quedaron bajo las aguas y cuyos residentes perdieron sus tierras de labor y de sustento, y fueron obligados a emigrar. Durante el intenso periodo de construcción de las obras, se creó un mercado de trabajo temporal que al cerrarse dejo "sin ingreso y con escasas oportunidades de reinsertarse en actividades agropecuarias a un número importante de personas" (Guillén, 2003). A la par, el establecimiento de actividades de la Comisión Federal de Electricidad y Petróleos Mexicanos atrajo trabajadores tanto de la entidad como de otros estados del país -en el caso de estos últimos especialmente personal calificado procedente de Veracruz, Tamaulipas y el Distrito Federal (Del Rey, 1998). En décadas más recientes, las ciudades de Tuxtla Gutiérrez, Tapachula, San Cristóbal de las Casas y Comitán de Domínguez se han consolidado como destinos urbanos para nuevos flujos migratorios. Como se concluye en una investigación reciente:

El reparto agrario, el incremento en las vías de comu-
nicación y la concentración de actividades económicas
en las ciudades han sido factores determinantes tanto
para el crecimiento como para la dirección que siguie-
ron los movimientos poblacionales en Chiapas duran-
te los últimos 35 años (Jáuregui y Ávila, 2007: 8).

Como territorio fronterizo, según se aprecia en el Cuadro 1, de las cuatro entidades limítrofes del sur de México, muy ampliamente Chiapas es la que tiene mayor colindancia longitudinal con Guatemala (654 kilómetros), por lo que su frontera dibuja una extensa línea de conexión y contención para la movilidad poblacional transfronteriza. Esa condición fronteriza fue hondamente acentuada como franja divisoria en los años de la guerra en Centroamérica (Kauffer, 2005), y en la actualidad es considerada por el gobierno estatal como "la puerta grande de la frontera sur". ${ }^{2}$ Con 4.3 millones de habitantes en el año 
2007, también es la entidad que concentra el mayor número de residentes de los cuatro estados sur fronterizos, cifra que representa poco más de la mitad (52.4\%) de la población total del conjunto de éstos.

\section{Cuadro 1. Población de las entidades fronterizas del sur de México 2006 y extensión territorial} colindante con Guatemala.

\begin{tabular}{|l|c|c|c|c||}
\hline Entidad & $\begin{array}{c}\text { Población } \\
\text { estatal }\end{array}$ & $\begin{array}{c}\text { Porcentaje } \\
\text { regional }\end{array}$ & $\begin{array}{c}\text { Frontera con } \\
\text { Guatemala (Km.) }\end{array}$ & $\begin{array}{c}\text { Porcentaje } \\
\text { regional }\end{array}$ \\
\hline \hline Chiapas & $4,363,413$ & 52.41 & 654 & 68.41 \\
\hline \hline Tabasco & $2,017,710$ & 24.23 & 112 & 11.72 \\
\hline \hline Quintana Roo & $1,175,475$ & 14.12 & $* 24$ & 2.51 \\
\hline \hline Campeche & 768,222 & 9.24 & 166 & 17.36 \\
\hline \hline Total regional & $8,324,820$ & 100.0 & 956 & 100 \\
\hline
\end{tabular}

* Quintana Roo colinda también con Belice en un extensión de 176 kilómetros.

Fuentes: CONAPO, Prontuario Demográfico de México 2007. DE: http://www.conapo.gob.mx/prontuario2007/prontuario. htm, y Chávez, 2007.

En la actualidad, Chiapas registra intensos flujos de ingreso y salida de personas, especialmente movimientos de carácter laboral. Por distintos puntos de la frontera internacional chiapaneca, ingresan y retornan trabajadores extranjeros, principalmente centroamericanos, que se emplean en labores agrícolas en la región del Soconusco y en la construcción, el comercio y los servicios en otras localidades de la entidad, particularmente en la ciudad de Tapachula; movilidad que se extiende hacia otros estados de la región sur mexicana. En años recientes se observa que la mayor proporción de los trabajadores transfronterizos son de origen guatemalteco, y en menores proporciones nativos de Honduras y El Salvador. Asimismo se observan en tránsito por la entidad individuos y familias que se dirigen hacia otras entidades mexicanas en busca de mejores oportunidades de vida y de empleo, al igual que quienes tienen como destino final los Estados Unidos. El mayor movimiento de ingreso y salida de estos flujos transfronterizos y de larga distancia ocurre por las localidades de Ciudad Hidalgo, Talismán y Ciudad Cuauhtémoc, importantes sitios de conexión por sus vías de comunicación terrestre con Centroamérica, el estado de Chiapas y el resto de México (EMIF-Guamex, 2006).

Respecto a los flujos de emigrantes, los chiapanecos han participado tanto en el sistema migratorio regional que conforman las entidades del sur del país, como en la migración nacional e internacional. En décadas recientes, la diversidad de actividades vinculadas con la industria extractiva petrolera en los estados de Campeche y Tabasco, así como aquellas otras derivadas de la intensa actividad turística en la Riviera Maya, y el crecimiento y expansión de los sectores comercial y de servicios en los centros urbanos de Cancún, Chetumal, Campeche y Villahermosa, han atraído trabajadores procedentes de Chiapas, y también de Centroamérica (Chávez et al., en prensa).

De acuerdo con los Resultados definitivos del il Conteo de Población y Vivienda 2005, entre los años 2000 y 2005, "llegaron a vivir" a Chiapas poco más de 24 mil personas, la mitad de ellos procedentes de sólo cuatro entidades mexicanas: Tabasco, Estado de México, Distrito Federal y Veracruz. En contraste, la misma fuente reportó que salieron de la entidad chiapaneca 90 mil personas, cuyos siete destinos principales en el interior del país fueron, en orden de participación porcentual, Baja California, Quintana Roo, Estado de México, Tabasco, Distrito Federal, Veracruz y Oaxaca; entidades cuyo conjunto sumó $60 \%$ del total de emigrantes chiapanecos en territorio nacional, según se detalla en el Cuadro 2.

Además de las cifras, la información del Cuadro 2 permite apreciar los circuitos migratorios en los que se mueven los chiapanecos. Uno de corta distancia localizado en las entidades del sur del país (Tabasco, Veracruz, Quintana Roo, Oaxaca). Otro de carácter tradicional en el ámbito nacional, localizado en el centro del país y conformado por el Distrito Federal y el Estado de México, que podría equipararse territorialmente con la enorme Zona Metropolitana de la Ciudad de México. 
El tercer circuito se extiende hacia la frontera norte mexicana, teniendo como destino primario el estado de Baja California y posiblemente articulándose allende la frontera hacia Estados Unidos.

Cuadro 2. Participación porcentual de inmigrantes y emigrantes, Chiapas 2000-2005.

\begin{tabular}{|l|c|l|c||}
\hline \multicolumn{2}{|c|}{ Inmigrantes } & \multicolumn{2}{c||}{ Emigrantes } \\
\hline \hline Tabasco & 14.4 & Baja California & 17.5 \\
\hline \hline E. México & 13.6 & Quintana Roo & 13.9 \\
\hline \hline Distrito Federal & 12.1 & E. México & 6.8 \\
\hline \hline Veracruz & 11.5 & Tabasco & 6.3 \\
\hline \hline Oaxaca & 9.7 & Distrito Federal & 5.7 \\
\hline \hline Baja California & 4.8 & Veracruz & 5.4 \\
\hline \hline Puebla & 4.7 & Oaxaca & 4.5 \\
\hline \hline $\begin{array}{l}\text { S u b t o t a I } \\
\text { porcentual }\end{array}$ & 70.8 & Subtotal porcentual & 60.1 \\
\hline \hline \begin{tabular}{l} 
Número total \\
\hline
\end{tabular}
\end{tabular}

Fuente: INEGI, Resultados definitivos del II Conteo de Población y Vivienda 2005.

Por una parte, vale la pena mencionar que entre los inmigrantes que llegaron a Baja California entre 2000 y 2005, los chiapanecos ocuparon el tercer sitio, sólo precedidos por sinaloenses y sonorenses; estos dos últimos, son flujos internos de histórica y constante participación migratoria en la entidad bajacaliforniana. Por otra parte, es relevante destacar que la ciudad de Tijuana recibió a la gran mayoría de esos inmigrantes chiapanecos, pues ocho de cada diez de ellos llegaron a residir a este municipio primordialmente urbano. De manera similar, los chiapanecos que emigraron a Quintana Roo se dirigían mayoritariamente (88\%) a sólo dos de sus ocho municipios: la mitad de ellos a Benito Juárez (donde se localiza Cancún) y cuatro de cada diez a Solidaridad, municipio donde se asienta Playa del Carmen (INEGI, 2005: disco compacto).
En relación con la condición de Chiapas como entidad de atracción migratoria transfronteriza, de acuerdo con el Instituto Nacional de Migración, anualmente se emplean en fincas cafetaleras, bananeras, cañeras y ganaderas mexicanas localizadas en la franja fronteriza con Guatemala, alrededor de 40 mil jornaleros agrícolas guatemaltecos, debidamente registrados y documentados por esa institución (INM, 2007: 6); cifra que no considera aquellos otros que laboraron sin ese tipo de autorización. En el año 2006, ese mismo Instituto informó que el mayor número de aseguramientos de extranjeros no autorizados se registró en las delegaciones regionales del estado de Chiapas, concentrando la entidad cerca de la mitad de su totalidad, según detalla el cuadro 3.

Cuadro 3. Aseguramientos registrados por el INM, 2006

\begin{tabular}{|l|c|c|}
\hline Delegaciones regionales & Enero-Diciembre & Porcentaje \\
\hline \hline Chiapas & 90,601 & 49.6 \\
\hline \hline Tabasco & 23.387 & 12.8 \\
\hline \hline Veracruz & 17,122 & 9.4 \\
\hline \hline Distrito Federal & 5,653 & 3.1 \\
\hline \hline Otros estados & 45,942 & 25.1 \\
\hline \hline Total & 182,705 & 100.0 \\
\hline
\end{tabular}

Fuente: Instituto Nacional de Migración, 2007: 7

Las cifras de trabajadores agrícolas documentados indican la importancia de la entidad como receptora de flujos laborales transfronterizos y a la vez ponen de manifiesto la intensa movilidad internacional de corta distancia que ocurre en el estado, representada no sólo por estos trabajadores sino también por otro tipo de visitantes y residentes de la región fronteriza de Guatemala con México, quienes se desplazan cotidianamente y por otros motivos (familiares o comer- 
ciales) entre las localidades vecinas de los dos países. Por su parte, los 90,601 aseguramientos de extranjeros no autorizados registrados en Chiapas durante el año 2006, revelan su posición como entidad de tránsito migratorio hacia otras regiones del país y hacia Estados Unidos, pues en ese mismo año el Instituto Nacional de Migración reportó que 400 mil migrantes que carecían de documentación migratoria trataron de atravesar el territorio nacional con destino al vecino país del norte (INM, 2007: 3).

La información hasta aquí sistematizada nos permite constatar que también en años recientes el estado de Chiapas ha sido un territorio de atracción migratoria para inmigrantes procedentes de entidades colindantes (Tabasco, Veracruz y Oaxaca), del centro del país (Estado de México, Distrito Federal y Puebla) y aun de la frontera norte (Baja California); asimismo y en forma numerosa y creciente para trabajadores agrícolas guatemaltecos. Simultáneamente, si bien la emigración de los chiapanecos ha sido característica y recurrente hacia destinos de corta travesía localizados en la región sur del país (especialmente los aledaños estados de Tabasco, Veracruz y Oaxaca) y hacia las dos entidades que en el ámbito nacional conforman la zona tradicionalmente receptora del centro del país (Distrito Federal y Estado de México), en años recientes se incorporaron progresiva y crecientemente a su movilidad territorial destinos de mayores distancias. Si bien en el sur del país, Quintana Roo ejerce una fuerte atracción migratoria por sus desarrollos turísticos, los chiapanecos se han aventurado de manera paulatina a itinerarios de mayor amplitud, especialmente hacia la frontera norte mexicana. De los 90 mil chiapanecos que emigraron del estado entre los años 2000 y 2005, cerca 16 mil se dirigían a Baja California, cifra que ubica a esa lejana entidad fronteriza del norte del país en el primer sitio como destino nacional de la emigración chiapaneca. Finalmente, en años recientes se registró un incremento del flujo de quienes pretendían emigrar a Estados Unidos, como expondremos a continuación.
Movilidad laboral en las fronteras: explorando dos encuestas de flujos migratorios

En colaboración con instituciones de los sectores público y académico, El Colegio de la Frontera Norte realiza el levantamiento de encuestas de flujos migratorios en las fronteras norte y sur de México. Estas fuentes de información permiten explorar las características sociodemográficas y la dinámica de los flujos que se desplazan por esas regiones, y aportar elementos para el análisis de su movilidad territorial.

Uno de los flujos laborales que llegan a la entidad chiapaneca es el de centroamericanos, mayoritariamente guatemaltecos, que se emplean en regiones y localidades chiapanecas, o bien que se dirigen a otros estados de México o se desplazan por la entidad en tránsito hacia los Estados Unidos, y que ingresan por diversidad de localidades ubicadas en la frontera entre Guatemala y México. El Mapa l permite ubicar geográficamente esa diversidad de puntos de internación y destacar que la gran mayoría se encuentran en la frontera chiapaneca.

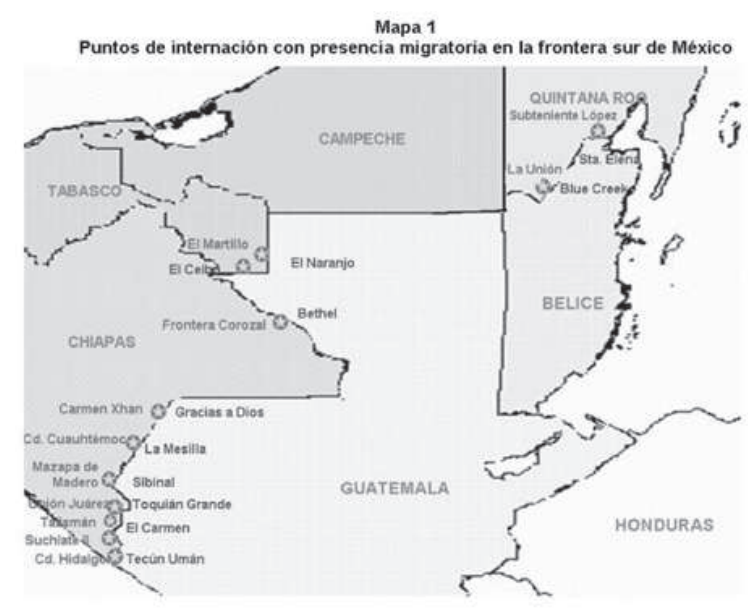

Fuente: INM, CONAPO, STPS, SRE, COLEF, Encuesta sobre migración en la frontera Guatemala-México 2004. México, 2006, p. 23. 
La Encuesta sobre migración en la frontera Guatemala-México (EMIF-GUAMEX), que capta el flujo de migrantes laborales guatemaltecos que se internaron por vía terrestre por la frontera de Chiapas hacia México, ${ }^{3}$ permite sustentar que alrededor de la mitad de esos movimientos no eran propiamente de "migrantes", sino más precisamente de trabajadores transfronterizos, pues durante los tres años de levantamiento continuo de información realizados de 2004 a 2006, entre 47\% y 53\% del conjunto manifestó que permanecerían en México sólo durante la jornada laboral diaria, retornando a sus lugares de origen en menos de 24 horas. Este comportamiento presenta tendencias diferenciales y contrastantes entre hombres y mujeres, pues en el conjunto femenino la estancia por sólo un día fue mayoritaria (alrededor del 70\%), mientras que entre los varones casi seis de cada diez se internaron para permanecer por más de un día. La misma fuente permite examinar el perfil sociodemográfico de estos trabajadores.

- Si bien en su gran mayoría eran varones, se observa una tendencia creciente en la participación femenina: 18\% en 2004 a 25\% en 2006.

- Sus edades se concentraron entre los 15 y 40 años, esto es, en la etapa más activa de la vida productiva; concentración característica de las migraciones laborales. Sin embargo, también los grupos de edad adquieren comportamientos diferenciales por sexo y tiempo de estancia en México. Los varones que permanecerían más de un día se ubicaron en edades más jóvenes pues la mitad de ellos eran menores de 30 años, mientras que la mayor concentración de mujeres ocurrió entre aquellas que permanecerían menos de un día y cuyas edades fluctuaban entre 20 y 39 años.

- Respecto al destino de los desplazamientos y los sectores de ocupación en esos destinos, la mayor proporción se dirigía a alguna finca, rancho o ejido en Chiapas y a las localidades de Tapachula y Ciudad Hidalgo, pretendiendo emplearse en actividades agrícolas cuatro de cada diez, y pro- porciones menores en el comercio y los servicios (tres y dos de cada diez, respectivamente). Este comportamiento también presentó variaciones por sexo y tiempo de estancia, pues entre los varones la mayoría de quienes se quedarían más de un día se dirigían al sector agropecuario y entre las mujeres a los servicios; mientras que hombres y mujeres que permanecerían menos de un día pretendían emplearse mayoritariamente en el comercio (formal e informal). En consecuencia, los empleos de hombres y mujeres en agricultura y servicios demandan estancias mayores a un día, mientras que quienes se dedicaban a actividades comerciales suelen ingresar y retornar el mismo día.

- Vale la pena mencionar que la mayoría de estos trabajadores guatemaltecos se internó en México utilizando un pase local o Forma migratoria de visitante local (FMVL), y a pesar de que cuatro de cada diez realizarían labores agrícolas, apenas uno de cada diez contaba con Forma migratoria de visitante agrícola (FMVA). Ello indica que las cifras de trabajadores agrícolas registradas oficialmente bajo esta forma (40 mil trabajadores anuales) subestiman la magnitud real de quienes laboran de hecho en esa actividad.

- Finalmente, la enorme mayoría de los entrevistados procedía de departamentos fronterizos guatemaltecos o cercanos a la frontera, $70 \%$ de ellos de San Marcos y 18\% de Quetzaltenango y sólo 6\% de departamentos no fronterizos, lo que corrobora la importancia de la región como espacio de movilidad de los flujos transfronterizos de guatemaltecos en Chiapas.

- En síntesis, la EMIF-GUAMEX nos permite apreciar dos flujos de trabajadores guatemaltecos que en proporciones más o menos similares se internaban por el sur de la entidad chiapaneca: aquellos que retornaban el mismo día y quienes permanecerían por más tiempo. En ambos flujos, la participación femenina presenta una tendencia 
creciente. Estos flujos transfronterizos de corta distancia que provienen masivamente de los departamentos fronterizos guatemaltecos de San Marcos y Quetzaltenango y circulan por Tecún Umán-Ciudad Hidalgo y El Carmen-Talismán y en menor magnitud por La Mesilla-Ciudad Cuauhtémoc, tienen mayoritariamente como destino sectorial y geográfico el trabajo agrícola en alguna finca, ejido o rancho en Chiapas, y en menor proporción el empleo (y posiblemente autoempleo) en actividades comerciales y de servicios en la región fronteriza y en las localidades de Tapachula y Ciudad Hidalgo.

- En dirección opuesta, la misma fuente nos permite examinar el flujo de migrantes guatemaltecos devueltos por las autoridades migratorias mexicanas. Respecto a ellos, vale la pena destacar lo siguiente:

- En los años 2004 y 2005, ocho de cada diez y en el año 2006 seis de cada diez ${ }^{4}$ se dirigían a los Estados Unidos, por lo que al ser detenidos y devueltos por las autoridades mexicanas, éstas resultaron ser un eficiente filtro que impidió su llegada e ingreso en el vecino país del norte; constituyéndose a la par el territorio mexicano en una frontera vertical muy extensa (Casillas, 2006).

- En los primeros dos años de levantamiento de la encuesta, cerca de la mitad de los entrevistados había permanecido la mayor parte del tiempo en Chiapas antes de ser detenidos y devueltos a Guatemala. Al sumar las otras entidades del sur del país (en orden de participación porcentual descendente: Veracruz, Tabasco, Oaxaca y Quintana Roo), la cifra alcanzó a siete de cada diez guatemaltecos detenidos y devueltos desde esa región. De 2004 a 2006, otras entidades mexicanas incrementaron su participación como lugares de mayor estancia de los guatemaltecos que se dirigían a los Estados Unidos transitando por México, pasando en conjunto de $13 \%$ a 27\% en ese lapso, cifra que indica, por una parte, la dispersión alcanzada en su movilidad y, por otra, la amplitud correlativa que han adquirido las detenciones por parte de las autoridades migratorias mexicanas en territorio nacional.

- A diferencia de los desplazamientos de los trabajadores transfronterizos, estos migrantes procedian en proporciones más equilibradas de departamentos guatemaltecos fronterizos (52\%) y no fronterizos (46\%). En términos de orígenes y destinos, ello permite advertir circuitos migratorios diferenciales entre los trabajadores transfronterizos y los migrantes internacionales guatemaltecos.

Respecto a los flujos migratorios de larga distancia, en años recientes se ha incrementado la participación de los chiapanecos en destinos como la frontera norte mexicana y los Estados Unidos. La Encuesta sobre migraciónen la frontera norte de México (EMIF-Norte) permite conocer la evolución de su participación en tres flujos: el que se desplaza del interior del país con destino a la frontera norte mexicana o los Estados Unidos (denominado en la encuesta flujo procedente del sur), los que retornan de manera voluntaria desde ese país o bien desde la frontera norte mexicana a sus entidades de origen en México (designado flujo procedente del norte que a su vez se divide en procedentes de Estados Unidos y procedentes de la frontera norte) y los que fueron devueltos por las autoridades migratorias estadounidenses. Para fines de este trabajo, exploramos la participación de los chiapanecos en dos de esos tres flujos, observando su creciente contribución al conjunto. ${ }^{5}$

La gráfica 1 permite apreciar la participación porcentual del flujo de emigrantes procedentes del sur ${ }^{6}$ que se desplazaban con destino a la frontera norte mexicana o hacia los Estados Unidos entre los años 1993 y 2006, y comparar el comportamiento de tres entidades de reconocida y larga tradición migratoria internacional hacia el vecino país del norte (Guanajuato, Michoacán y Jalisco) con otras tres de nueva participación: Chiapas, Veracruz y el Distrito Federal. 


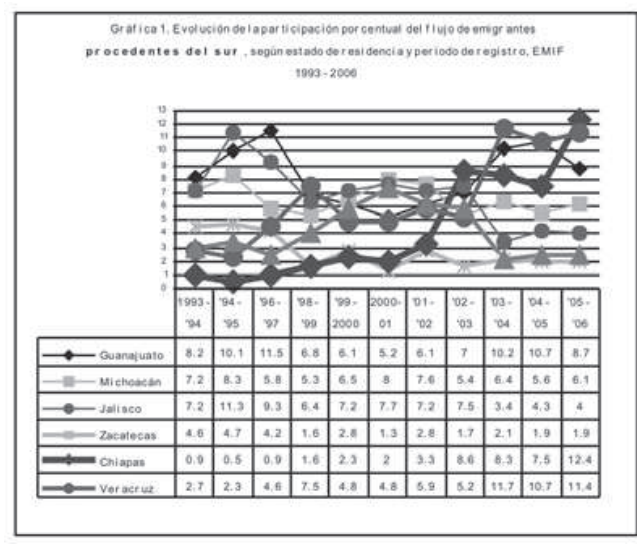

En la gráfica se observa que Guanajuato, Michoacán y Jalisco experimentaron un descenso en su contribución al flujo de emigrantes que se desplazaban por la frontera norte con destino a esa región o al vecino país del norte, aunque Guanajuato parece recuperar su movilidad al final del periodo registrado en la gráfica. El descenso en la movilidad del flujo de migrantes de esas entidades pudiera estar relacionado con el incremento de la vigilancia fronteriza que el gobierno estadounidense desplegó en su frontera con México con objeto de desalentar y dificultar el ingreso no documentado, y que inició en el año 1993 y se extendió en años subsecuentes a lo largo de toda la frontera. La información que se difunde a través de las redes sociales de los residentes de comunidades de larga tradición migratoria permite a sus migrantes (experimentados y primerizos) emprender y desarrollar estrategias alternativas frente a los nuevos obstáculos establecidos para su movilidad (Anguiano y Trejo, 2007); entre ellas, evitar ingresos y retornos recurrentes anuales y prolongar la estancia en el origen o el destino, por lo que sus movimientos migratorios decrecen y, en consecuencia, la participación de estas entidades en los flujos migratorios que capta la EMIF-Norte reducen sus proporciones. ${ }^{7}$

En contraste, llama la atención la creciente participación de tres entidades de nueva incorporación a los flujos que se dirigen a la frontera norte y los Estados Unidos, especialmente en condiciones en que el ingreso al vecino país ha sido progresivamente dificultado. Veracruz y el Distrito Federal muestran una participación creciente y expansiva que, en el caso del primero, inicia en 1994 y se extiende hasta 1999, tiende a estabilizarse en años posteriores y se incrementa notoriamente entre 2003 y 2006; mientras que la participación del segundo asciende de manera continua entre los años 1996 y 2001, experimenta un ligero descenso a partir de ese año y cae en el último trienio registrado en la gráfica, comportamiento posiblemente asociado a los acontecimientos de septiembre 11 del 2001 y a las sucesivas medidas de seguridad fronteriza estadounidense vinculadas con los atentados terroristas. En el caso de Chiapas, si bien su participación se mantiene en pequeñas proporciones y experimenta un crecimiento lento por varios años, sorprende que sea precisamente a partir del 2001 cuando su contribución asciende súbitamente, y alcanza la mayor participación porcentual del conjunto en el último año registrado en la gráfica. Aventurando un par de hipótesis sobre este comportamiento, por una parte postularía que la mayor proporción de esos emigrantes chiapanecos se dirigía a la frontera norte; por otra, que quienes se dirigían a los Estados Unidos respondían a, y a la vez revelaban, nuevas formas de reclutamiento focalizado y desplegado en localidades, entidades y regiones expulsoras de migrantes de reciente integración a ese flujo internacional. Un dato adicional que apoyaría el reclutamiento focalizado en la entidad es el incremento de servicios de transporte foráneo que se desplazan desde Chiapas hasta la frontera norte mexicana (Villafuerte y García, 2004, García et al., 2007). 8

De manera similar, la gráfica 2 permite examinar el comportamiento del flujo de migrantes no documentados y devueltos por las autoridades estadounidenses. Podemos decir que se trata de un conjunto de migrantes internacionales no exitosos, pues fueron capturados y devueltos. En primer término, observamos que Guanajuato y Jalisco mantienen proporciones ligeramente variables 
con tendencia a la baja el primero y a una ligera alza el segundo, y Michoacán muestra un ascenso un poco más pronunciado que fluctúa entre dos y tres puntos porcentuales. Esto significa que estas tres entidades continúan manteniendo su aportación al flujo internacional, aun entre los migrantes no autorizados y devueltos.

Por su parte, Veracruz y Chiapas, estados de reciente participación en el flujo migratorio internacional, muestran notorios ascensos en su participación en el conjunto, al igual que Oaxaca. Llama la atención que en el último periodo fue precisamente Chiapas la entidad que aportó la mayor proporción a este flujo, lo que indica que su población se está integrando crecientemente al flujo internacional, pero que sus migrantes son los menos exitosos tratando de internarse sin documentos eintentando permanecer en el vecino país. Entre los migrantes que en años recientes han sufrido las mayores tragedias tratando de cruzar la frontera hacia Estados Unidos en forma encubierta o subrepticiamente, los chiapanecos ocupan las primeras posiciones. De acuerdo con el Programa de Repatriación voluntaria instrumentado a partir de 2004 en la desértica frontera de Sonora-Arizona por los gobiernos mexicano y estadounidense, entre los migrantes rescatados y devueltos a México, los chiapanecos ocupan los primeros sitios, sólo superados por veracruzanos y poblanos y sumados a otros migrantes procedentes de los estados de México, Guerrero, Oaxaca y Michoacán.

Grafica 2 Evolucióndela participaciónporcentual del flujode emigrant es devuelt os, sogunestadoderesdencia periododeregistro, EMIF 1993.

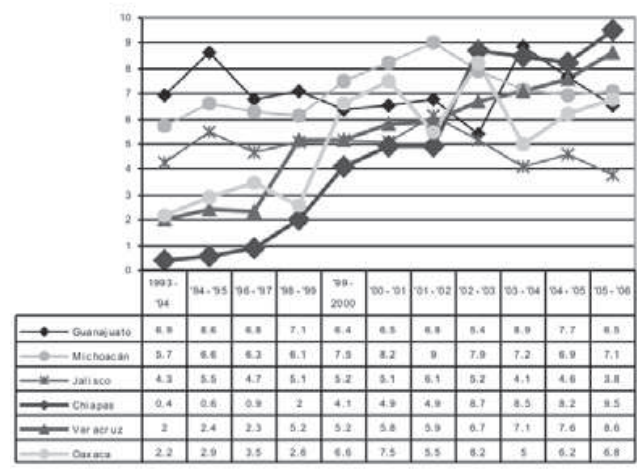

La información hasta aquí sistematizada nos permite postular que en años recientes, al igual que ocurre en el país en su conjunto pero posiblemente con mayor intensidad y velocidad, Chiapas se está conformando como un territorio de inmigración, emigración y tránsito migratorio. Esta condición deriva en importantes retos para la política pública, especialmente para la política de población en sus aspectos migratorio y laboral, tanto para el país como para la entidad y, en consecuencia, en retos y tareas para la cooperación intergubernamental.

\section{Reflexiones finales}

Territorialmente, Chiapas forma parte de la frontera sur de México que colinda con la frontera este de Guatemala y por extensión con Centroamérica. En su calidad de territorio fronterizo del sur del país y área económica estratégica por la explotación de sus recursos naturales, en Chiapas se ha desarrollado mercados de trabajo que emplean tanto a nativos como a inmigrantes internos e internacionales. A pesar de ello, no se ha logrado generar empleo en cantidades y calidades suficientes y adecuadas para que los chiapanecos permanezcan en la entidad, pues sus movimientos migratorios de corto recorrido al interior del estado y escasas magnitudes al exterior del mismo, en años recientes se han ampliado e incrementado a destinos de mayor distancia, entre ellos, los desarrollos turísticos de la Península de Yucatán, las localidades urbanas fronterizas del norte de México y masiva y crecientemente a los Estados Unidos.

En dos trabajos presentados como tesis de doctorado en los años 1999 y 2001, ${ }^{9}$ sus autores se preguntaban por qué los chiapanecos, a diferencia de otros grupos étnicos como los oaxaqueños, no emigraban en busca de oportunidades laborales fuera de la entidad. En menos de una década, esa situación parece estar modificándose aceleradamente pues la emigración chiapaneca se ha incrementado de manera notoria y masiva, extendiendo sus destinos primarios en la Riviera Maya, la frontera norte mexicana y los Estados Unidos hacia nuevas geografías 
en el interior y exterior del país.

Como territorio de intensa movilidad transfronteriza, Chiapas enfrenta el reto de participar en la gestión y regulación que pretende establecer el estado mexicano a través del Instituto Nacional de Migración a partir de su Propuesta de política migratoria integral en la frontera sur, a través de atendere incorporar en forma legal, segura y ordenada los flujos laborales, principalmente de guatemaltecos y de otros centroamericanos que llegan a la entidad, así como reconocer su aportación tanto a la economía y sociedad chiapanecas como al país en su conjunto, y actuar en consecuencia con respeto a sus derechos laborales y humanos.

Respecto a la emigración de sus habitantes, el reto es doble. Por una parte, la creación de empleos dignos en cantidades y calidades suficientes que permitan emplear a su población en su territorio, y considerar a la emigración una más de las posibles opciones laborales y no un imperativo extremo para la supervivencia y menos aún la única, última y más difícil elección. Por otra parte, el reto de imaginar, crear y desarrollar una política migratoria para apoyar a aquellos que deciden emigrar, tanto al interior como al exterior del país. Ambos retos, lo son también para México como nación.

Finalmente, como territorio de inmigración nacional y extranjera, en Chiapas - al igual que en todo el paíscontinúa siendo un reto el respeto a la diversidad cultural, una más de las riquezas de su ancestral población - la de Chiapas y la del país-; más aún, en un mundo en que la homogenización de las ideas y de la cultura propiciada por "LaMcDonalización de la sociedad" —refiriendo a George Ritzer - impele de manera equivocada hacia la supresión de esa diversidad y niega obtusamente las posibilidades de las sociedades multiétnicas, como de hecho lo son Chiapas y el país.

\section{Notas}

${ }^{1}$ Según informó el Banco de México, en el año 2007, Chiapas recibió 815 millones de dólares por concepto de remesas, cifra que según estimaciones del gobierno de esa entidad representaba "93\% del total generado por la masa salarial del sector formal en Chiapas". Asimismo, el gobierno estatal destacaba que se habían expedido 300 mil matrículas consulares a igual número de chiapanecos en Estados Unidos. Cf. "Política migratoria del Gobierno de Chiapas", en Chiapas. Enlace con el Mundo. Tuxtla Gutiérrez, Gobierno del Estado de Chiapas, Coordinación de Relaciones Internacionales, mayo 2008, núm. 28, p. 13. Más allá de deliberar sobre la exactitud de estas cifras, lo que interesa destacar es la relevancia y visibilidad que el fenómeno migratorio ha adquirido en la entidad.

2 "Expone gobernador Sabines políticas migratorias de su gobierno ante relator de la ONU, Jorge Bustamante", Chiapas. Enlace con el Mundo. Tuxtla Gutiérrez, Gobierno del Estado de Chiapas, Coordinación de Relaciones Internacionales, mayo 2008, núm. 28, pp. 11-12.

${ }^{3}$ Se refiere al conjunto de personas entrevistadas nacidas en Guatemala que tenían 15 años y más de edad, cuyo motivo del desplazamiento e ingreso a México fue trabajar o buscar trabajo y que se internaron al país por las localidades chiapanecas de Ciudad Hidalgo, Talismán y Ciudad Cuauhtémoc. Cf. EMIFGUAMEX 2004.

${ }^{4}$ Proporción que puede haber disminuido ese último año por efecto de los estragos causados por el Huracán Stan que asoló la región en octubre de 2005.

${ }^{5}$ Excluimos del análisis el flujo procedente del norte debido a que su magnitud numérica limita las inferencias posibles.

'La encuesta define como "flujo procedente del SUR" a personas mayores de 12 años, no nacidas en Estados Unidos ni residentes de las localidades fronterizas o de Estados Unidos, que llegan a las ciudades fronterizas donde se levanta la encuesta sin una fecha comprometida de retorno y cuya estancia se debe a motivos de trabajo, cambio de residencia o en tránsito hacia el norte, o bien por razones de estudio, paseo o visita a familiares o amigos. ${ }^{7}$ Es el caso del estado de Zacatecas, que cuenta con una amplia red de asociaciones de migrantes constituidos en Federaciones con presencia en 10 entidades de la Unión Americana que se constituyen en canales y redes de comunicación que transmiten información oportuna

152 Revista LiminaR. Estudios sociales y humanísticos, año 6, vol. VI, núm. 2, diciembre de 2008, Tuxtla Gutiérrez, Chiapas. ISSN: 1665-8027 
que incentiva o inhibe la movilidad migratoria.

${ }^{8}$ En la ciudad de San Cristóbal de Las Casas pudimos observar la diversidad de itinerarios de la línea de autotransportes Chiapas-Tijuana. De igual forma, en la localidad de Altar, Sonora, hay autotransportes foráneos que arriban desde distintas localidades chiapanecas y retornan a ellas.

${ }^{9}$ El primero presentado por Germán Martínez Velasco en El Colegio de la Frontera Norte, el segundo por Jennifer Balkan en la Universidad de Texas en Austin (véase bibliografía).

\section{Bibliografía}

Anguiano, María Eugenia y Alma Trejo Peña, 2007, "Políticas de seguridad fronteriza y nuevas rutas de movilidad de migrantes mexicanos y guatemaltecos", en LiminaR. Estudios sociales y humanísticos, año 5, vol. V, núm. 2, diciembre, pp.47-65.

Balkan, Jennifer Lynn, 2001, Why Not Migrate? A case Study of Two Rural Villages in Chiapas, México. Tesis de doctorado. Austin, Universidad de Texas.

Burke, Garance, 2005, "Yucatecos and Chiapanecos in San Francisco: Mayan Immigrants from New Communities", en Jonathan Fox and Gaspar Rivera-Salgado (editors), Indigenous Mexican Migrants in the United States. San Diego, University of California, pp. 343-354.

Cruz Burguete, Jorge Luis y Gabriela Patricia Robledo Hernández, 2001, "Cambio social y movimientos de población en la región fronteriza de Chiapas”, en Convergencia, año 8, núm. 26, pp. 33-53

Casillas R., Rodolfo, 2006, Una vida discreta, fugazy anónima: los centroamericanos transmigrantes en México. México, SEGOB-Conacyt, http://www.insyde.org.mx/IMG/ pdf_RodolfoCasillasUnavidadiscretafugazyanonima. pdf. Consultado Mayo 15, 2007.

—, 2008, Mesoamérica. El Sur Mexicano y Centroamérica, Fortalezas y Debilidades. México, Consejo Mexicano de Asuntos Internacionales, Cuadernos 4, Septiembre.

Castillo, Manuel Ángel, 1994, "La migración internacional y el problema de los refugiados", en Políticas de población en Centroamérica, El Caribe y México. México, INAP-UNAM-PROLAP.

Castillo, Manuel Ángel, 2003, "Mexico-Guatemala Border: New Control on Transborder Migration in View of Recent Integration Schemes", en Frontera Norte, vol. 15, núm. 29, enero-junio, pp. 35-65.

Consejo Nacional de Población, 2000, Migración internacional en la frontera sur de México. Boletín, año 4, núm. 12, $16 \mathrm{pp}$.

Consejo Nacional de Población, Índices de Marginación, 2005, http://www.conapo.gob.mx/publicaciones/indice2005.htm.

Consejo Nacional de Población, La situación demográfica de México 2006, http://www.conapo.gob.mx/publicaciones/sdm2006/SDM2006.pdf.

Chávez, Ana María et al., Tendencias del crecimiento económico, del empleo y de la migración interna e internacional en las regiones Sur, Golfo y Península de Yucatán: Un estudio comparativo. México, CONACYT, en prensa.

Del Rey Poveda, Alberto, 1998, "Las expulsiones y los desplazamientos en las comunidades indígenas de Los Altos de Chiapas: consecuencias no anticipadas de la modernización", en América Latina Hoy, año/vol. 19, julio, p. 13-21.

Didou Aupetit Sylvie, 2003, "Producción sobre movimiento social en las revistas de Ciencias Sociales en Chiapas: algunos comentarios", en LiminaR. Estudios sociales y humanísticos, año 1, vol. 1, núm. 1, junio, pp. 109-119.

Fortuny Loter de Mola, Patricia y Mirian Solís Lizama, 2006, "Solidaridades entre poblaciones móviles: campesinos, mestizos e indígenas mexicanos en el Suroeste de La Florida”, en Desacatos, núm. 20, eneroabril, pp. 135-154.

García Aguilar, María del Carmen y María Tarrío García, 2006, "Migración internacional y derechos humanos, los transmigrantes centroamericanos en la frontera sur de México", en Carlos Miranda Videgaray et al. (coordinadores), Los nuevos rostros de la migración en el mundo. México, OIM-INM-Gobierno de Chiapas, pp. 195-220. 
García Sosa, Juan Carlos et al., 2007, "Migración internacional indígena y vulnerabilidad ante el VIH-SIDA. Los Chamulas entre las barras y las estrellas", en LiminaR. Estudios sociales y humanísticos, año 5, vol. V, núm. 1 , junio, pp. 119-144.

Guillén, Diana, 2003, "Redimensionamiento de una frontera largamente olvidada: Chiapas 1973-1993”, en Frontera Norte, vol. 15, núm. 30, julio-diciembre, pp. 121-149.

INEGI, Resultados definitivos del II Conteo de Población y Vivienda 2005, http:/www.inegi.gob.mx/inegi/contenidos/espanol/prensa/Boletines/Boletin/Comunicados/ Especiales/2006/Mayo/comunicall.pdf.

INEGI, II Conteo de Población y Vivienda 2005. Resultados definitivos. Estados Unidos Mexicanos y entidades federativas, disco compacto.

Instituto Nacional de Migración, 2005, Propuesta de política migratoria integral en la frontera sur de México. México, INM.

Instituto Nacional de Migración, 2007, Revista INM. Año 5, núm. 3, marzo.

INM, CONAPO, COLEF, SEGOB, STPS y SRE, 2006 , Encuesta sobre Migración en la frontera Guatemala-México 2004. México.

Jáuregui Díaz, José Alfredo y María de Jesús Ávila Sánchez, 2007, "Estados Unidos, lugar de destino para los migrantes chiapanecos”, en Migraciones Internacionales. Vol. 4, núm. l (12), enero-junio, pp. 5-38

Kauffer Michel, Edith F., 2005, "De la frontera política a las fronteras étnicas: refugiados guatemaltecos en México", en Frontera Norte, Vol. 17, núm. 34, julio-diciembre, pp. 7-36.

Marc-henry, André, 2004, "La frontera olvidada de México: Una zona de alto riesgo", en OIM Noticias. Ginebra, Organización Internacional para las Migraciones, Junio, pp. 8-9.

Martínez Velasco, Reynaldo Germán, 1999, ¿Por quéquedarse en Chiapas? Un análisis de los factores de la migración intraestatal de la población indígena. Tesis de doctorado en Ciencias Sociales, Tijuana, El Colegio de la Frontera Norte,.

Martínez Velasco, Germán, 2005, "Conflicto étnico y mi- graciones forzadas en Chiapas", en Política y Cultura, núm. 23, pp. 195-210.

Morales Bermúdez, Jesús, 2003, "Las revistas de Ciencias Sociales en Chiapas: los años noventa", en LiminaR. Estudios sociales y humanísticos, año 1, vol. 1, núm. 1, junio, pp. 120-125.

Olivera Bustamante, Mercedes, 2004, "Subordinación de género e interculturalidad. Mujeres desplazadas en Chiapas", en LiminaR. Estudios sociales y humanísticos, año 2, vol. II, núm. 1, junio, pp. 25-49.

Ortiz Millán, Óscar Hugo, "Características e implicaciones de los trabajadores guatemaltecos en Chiapas, un tema para la política migratoria”, en María Eugenia Anguiano y Rodolfo Corona Vázquez (coordinadores), Flujos migratorios en la frontera Guatemala-México, México, COLEF-INM, en prensa.

Peña Piña, Joaquín et al., 2000, "Determinantes socioeconómicos de la migración laboral: el caso de los indígenas mames de la Sierra Madre de Chiapas, México", en Papeles de Población, año 6, núm. 23, enero-marzo, pp. 153-179.

Peña Piña, Joaquín, 2005, "Las políticas del estado, cambio social y migración laboral", en Política y Cultura, núm. 23, pp. 25-42.

SRE, SEGOB-INM, Programa Repatriación Voluntaria al Interior 2005, http://www.sre.gob.mx/eventos/repatriacion/ $\$ 88$.

Tovar González, Ma. Elena, "Extranjeros en el Soconusco", en Revista de Humanidades: Tecnológico de Monterrey, núm. 8, pp. 29-43.

Villafuerte Solís, Daniel y María del Carmen García Aguilar, 2004, "Pobreza y migración en la Sierra de Chiapas”, en LiminaR. Estudios sociales y humanísticos, año 2, vol. II, núm. 1, junio, pp. 81-93.

Villafuerte Solís, Daniel, 2003, “Chiapas: las fronteras del desarrollo”, en LiminaR. Estudios sociales y humanísticos, año 1, vol. 1, núm. 1, junio, pp. 69-98.

- 2007, "La frontera sur de México en tiempos de globalización”, en Emmanuel Brunet-Jailly (editor), Borderlands. Comparing Border Security in North America and Europe, Quebec, University of Ottawa Press. 